\title{
A semi-classical over-barrier model for charge exchange between highly charged ions and one--optical electron atoms
}

\author{
Fabio Sattin * \\ Consorzio RFX, Corso Stati Uniti 4, 35127 Padova, ITALY
}

\begin{abstract}
Absolute total cross sections for electron capture between slow, highly charged ions and alkali targets have been recently measured. It is found that these cross sections follow a scaling law with the projectile charge which is different from the one previously proposed basing on a classical over-barrier model $(\mathrm{OBM})$ and verified using rare gases and molecules as targets. In this paper we develop a "semi-classical" (i.e. including some quantal features) OBM attempting to recover experimental results. The method is then applied to ion-hydrogen collisions and compared with the result of a sophisticated quantum-mechanical calculation. In the former case the accordance is very good, while in the latter one no so satisfactory results are found. A qualitative explanation for the discrepancies is attempted.
\end{abstract}

PACS numbers: $34.70+\mathrm{e}, 34.10 .+\mathrm{x}$

\section{INTRODUCTION}

The electron capture processes in collisions of slow, highly charged ions with neutral atoms and molecules are of great importance not only in basic atomic physics but also in applied fields such as fusion plasmas and astrophysics.

In the past years a number of measurements have been carried on the collisions between highly charged ions and rare gases [1] or molecules [2], in which one or several electrons were transferred from the neutral target to a charged projectile:

$$
A^{+q}+B \rightarrow A^{(q-j)+}+B^{j+}
$$

Their results-together with those from a number of other laboratories-yielded a curve which can be fitted within a single scaling law (a linear relationship) when plotting cross section $\sigma$ versus projectile charge $q$ : it is almost independent of the projectile species and of the impact velocity $v$ (at least in the low-speed range $v<1 \mathrm{au}$ ). When one extends experiments to different target species, the same linear relation holds between $\sigma$ and $q / I_{t}^{2}$, with $I_{t}$ the ionization potential of the target 3, 4 .

It is found that this scaling law could to be predicted, in the limit of very high projectile charge, by a modification of an extended classical over-barrier model (ECBM), allowing for multiple electron capture, proposed by Niehaus [5]. Quite recently a confirmation of this scaling has come from a sophisticated quantum-mechanical calculation [6].

Similar experiments were carried on more recently for collisions between ions and alkali atoms 17. The results show that the linear trend is roughly satisfied, but the slope of the straight line is grossly overestimated by the ECBM: in Fig. 1 we show some data points (stars with error bars) together with the analytical curve from the ECBM (dashed curve) which, for one-electron atoms, is written [3, 4 ]

$$
\sigma=2.6 \times 10^{3} q / I_{t}^{2} \quad\left[10^{-20} \mathrm{~m}^{2}\right]
$$

$\left(I_{t}\right.$ in $\left.\mathrm{eV}\right)$. It should be noticed that experimental data are instead well fitted by the results of a Classical Trajectory MonteCarlo (CTMC) code 7.

The ECBM of ref. [3] works in a simplified one-dimensional geometry where the only physically meaningful spatial dimension is along the internuclear axis. It does not take into account the fact that the electrons move in a three-dimensional space. This means that only a fraction of the electrons actually can fulfil the conditions dictated by the model. For rare gases and molecules, which have a large number of active electrons, this can be not a trouble (i.e., there are nearly always one or more

\footnotetext{
*E-mail: sattin@igi.pd.cnr.it
} 
electrons which can participate to the collision). For alkali atoms with only one active electron, on the other hand, an overestimate of the capture probability by OBM's could be foreseen.

With present-days supercomputers there are relatively few difficulties in computing cross sections from numerical integration of the time-dependent Schrödinger equation (e.g. refer to ref. [6]). Notwithstanding this, simple models are still valuable since they allow to get analytical estimates which are easy to adapt to particular cases, and give physical insight on the features of the problem. For this reason new models are being still developed 80.9].

In this paper we present a modified OBM which allows to get a better agreement with the experimental data of ref. [7].

\section{THE MODEL}

We start from the same approach as Ostrovsky 8 (see also [10 ) : be $\mathbf{r}$ the electron vector relative to the neutral atom $(\mathbf{T})$ and $\mathbf{R}$ the internuclear vector between $\mathbf{T}$ and the projectile $\mathbf{P}$ (see Fig. 2 for a picture of the geometry: it is an adaptation of Figure 1 from ref. 8 ). Let us consider the plane containing the electron, $\mathbf{P}$ and $\mathbf{T}$, and use cylindrical polar coordinates $(\rho, z, \phi)$ to describe the position of the electron within this plane. We can choose the angle $\phi=0$ and the $z$ direction along the internuclear axis. We will assume that the target atom can be described as an hydrogenlike atom, which is not a bad approximation when dealing with alkali atoms.

The total energy of the electron is

$$
E=\frac{p^{2}}{2}+U=\frac{p^{2}}{2}-\frac{Z_{t}}{\sqrt{\rho^{2}+z^{2}}}-\frac{Z_{p}}{\sqrt{\rho^{2}+(R-z)^{2}}} .
$$

$Z_{p}$ and $Z_{t}$ are the charge of the projectile and the effective charge of the target seen by the electron, respectively, and we are using atomic units.

We can also approximate $E$ as

$$
E(R)=-E_{n}-\frac{Z_{p}}{R}
$$

$E_{n}$ is given by the quantum-mechanical value: $E_{n}=Z_{t}^{2} /\left(2 n^{2}\right)$. This expression is asimptotically correct as $R \rightarrow \infty$.

On the plane $(\mathrm{e}, \mathbf{P}, \mathbf{T})$ we can draw a section of the equipotential surface

$$
U(z, \rho, R)=-E_{n}-\frac{Z_{p}}{R} .
$$

This represents the limit of the region classically allowed to the electron. When $R \rightarrow \infty$ this region is decomposed into two disconnected circles centered around each of the two nuclei. Initial conditions determine which of the two regions actually the electron lives in.

As $R$ diminishes there can be eventually a time where the two regions become connected. It is easy to solve eq. (5) for $R$ by imposing that $\rho_{m}=0$ and that there must be an unique solution for $z$ with $0<z<R$ :

$$
R_{m}=\frac{Z_{t}+2 \sqrt{Z_{t} Z_{p}}}{E_{n}}
$$

In the spirit of OBMs it is the opening of the equipotential curve between $\mathbf{P}$ and $\mathbf{T}$ which leads to a leakage of electrons from one nucleus to another, and therefore to charge exchange. Along the internuclear axis the potential $U$ has a maximum at

$$
z=z_{0}=R \frac{\sqrt{Z_{t}}}{\sqrt{Z_{p}}+\sqrt{Z_{t}}} .
$$

Whether the electron crosses this potential barrier depends upon its initial conditions. These are chosen from a statistical ensemble, which we will leave unspecified for the moment. Let $N_{\Omega}$ be the fraction of trajectories which lead to electron loss at the time $t$ and $W(t)$ the probability for the electron to be still bound to the target, always at time $t$. The fraction of losses in the interval $t, t+d t$ is given by

$$
d W(t)=-N_{\Omega} \frac{d t}{T_{e m}} W(t)
$$


with $T_{e m}$ the period of the electron motion along its orbit. A simple integration yields the leakage probability

$$
P_{l}=1-\exp \left(-\frac{1}{T_{e m}} \int_{-\infty}^{+\infty} N_{\Omega} d t\right) .
$$

In order to actually integrate Eq. (9) we need to know the collision trajectory; an unperturbed straight line with $b$ impact parameter is assumed:

$$
R=\sqrt{b^{2}+(v t)^{2}}
$$

At this point it is necessary to give an explicit expression for $N_{\Omega}$. The electron is supposed to be in the ground state $(n=1, l=m=0) . T_{\text {em }}$ becomes therefore [11]

$$
T_{e m}=2 \pi / Z_{t}^{3}
$$

Ref. [8] adopts a geometrical reasoning: the classical electron trajectories, with zero angular momentum, are ellipses squeezed onto the target nucleus. The only trajectories which are allowed to escape are those whose aphelia are directed towards the opening within the angle $\pm \theta_{m}$. The integration over this angle yields an analytical expression for $N_{\Omega}$ (Eq. 17 of ref. [8]). In Fig. 1 we show the results obtained using Ostrovsky's model ( dotted curve-eqns. 8,17 of ref. 81) 17. Notice that from direct inspection of the analytical formula, one sees that the scaling law is not exactly satisfied, at least at small values of the parameter $q / I_{t}^{2}$, and this is clearly visible in the plot. The result is almost equal to the scaling (2).

The present approach is based on the electron position instead than on electron direction. The recipe used here is (I) to neglect the dependence from the angle: all electrons have the same probability of escaping, regardless of their initial phase. Instead, (II) the lost electrons are precisely those which, when picked up from the statistical ensemble, are found farther from nucleus $\mathbf{T}$ than the distance $z_{0}$ :

$$
N_{\Omega}=\int_{z_{0}}^{\infty} f(r) d r
$$

with $f(r)$ the electron distribution function.

There is not a unique choice for $f(r)$ : the (phase-space) microcanonical distribution

$$
\tilde{f}(\mathbf{r}, \mathbf{p}) \propto \delta\left(E_{n}+\frac{p^{2}}{2}-\frac{Z_{t}}{r}\right)
$$

( $\delta$ is the Dirac delta) has been often used in literature since the works [12] as it is known that, when integrated over spatial coordinates, it reproduces the correct quantum-mechanical momentum distribution function for the case of the electron in the ground state 13. (more recently the same formalism has been extended to Rydberg atoms 14]). After integration over momentum variables one gets instead a spatial distribution function [15]

$$
f_{m c}(r)=\frac{Z_{t}\left(2 Z_{t}\right)^{3 / 2}}{\pi} r^{2} \sqrt{\frac{1}{r}-\frac{Z_{t}}{2}}, \quad r<2 / Z_{t}
$$

and zero elsewhere (The lowerscript "mc" is to emphasize that it is obtained from the microcanonical distribution). However, this choice was found to give poor results. It could be expected on the basis of the fact that (14) does not extend beyond $r=2 / Z_{t}$ and misses therefore all the large impactparameter collisions. In the spirit of the present approach, it should be instead important to have an accurate representation of the spatial distribution. We use therefore for $f(r)$ the quantum mechanical formula for an electron in the ground state:

$$
f_{1 s}(r)=4 Z_{t}^{3} r^{2} \exp \left(-2 Z_{t} r\right)
$$

which, when substituted in (12), gives

\footnotetext{
${ }^{1}$ Beware of a small difference in notation between the present paper and [8: here we use an effective charge for the target, $Z_{t}=\sqrt{2 E_{n}}$, while [8] uses an effective quantum number $n_{t}=1 / \sqrt{2 E_{n}}$ with the effective charge of the target set to 1 .
} 


$$
N_{\Omega}=\left[1+2 z_{0} Z_{t}+2\left(z_{0} Z_{t}\right)^{2}\right] \exp \left(-2 z_{0} Z_{t}\right)
$$

Since the choice for $f(r)$ does not derive from any classical consideration, we call this method a "semi-classical" OBM.

Notice that, in principle, one could go further and compute $f(r)$ from a very accurate wavefunction, fruit of quantum mechanical computations (see [16]), but this is beyond the purpose of the present paper (it could be worthy mentioning a number of other attempts of building stationary distributions $f(r)$, mainly in connections with CTMC studies, see [17 19]).

The $f(r)$ of Eq. (15) does not reproduce the correct momentum distribution, nor the correct energy distribution (which could be obtained only by using eq. 113). However, it is shown in 15 that this choice gives an energy distribution for the electrons, $f(E)$, peaked around the correct value $E_{n}$, and $<E\rangle=E_{n}$, where $\langle\ldots\rangle$ is the average over $f(E)$.

Some important remarks are to be done here. First of all, a question to be answered is: why use an unperturbed distribution, when the correct one should be sensitively modified by the approaching of the projectile. The answer is, obviously, that this choice allows to perform calculations analitically. We are doing here a sort of classical counterpart of a quantum-mechanical Born calculation: there, too, the matrix elements are computed as scalar products over unperturbed states, regardless of any perturbation induced by the projectile. In the following, however, some considerations about possible improvements over this simple approximation will be done.

A second question regards the meaning of the factor $d t / T_{e m}$ in eq. (\$): in Ostrovsky's paper this is the fraction of electrons which enter the loss zone during the time interval $d t$ and is valid under the hypothesis of a uniform distribution of initial phases of the electrons. In our case this this assumption ceases to be valid: electrons actually spend different fractions of their time at different radial distances from $\mathbf{T}$, depending on their energy. We will do a (hopefully not too severe) assumption by assuming that, on the average, the expression (8) still holds.

\section{RESULTS}

\section{A. Iodine - Cesium}

This study has been prompted by the ion-atom experiments of [7]: first of all, therefore, we apply the above model to the process of electron capture

$$
\mathrm{I}^{q+}+\mathrm{Cs} \rightarrow \mathrm{I}^{(q-1)+}+\mathrm{Cs}^{+}
$$

with $q=6 \div 30$. Impact energy is $1.5 \times q \mathrm{keV}$ [7]. The ionization potential of Cesium is $I_{t}=3.9$ $\mathrm{eV}$. Solid line in Fig. 1 is the result of the present model: the agreement is fairly good.

\section{B. Bare ions - Hydrogen}

As second test, we have computed cross section for captures

$$
\mathrm{H}+\mathrm{O}^{8+} \rightarrow \mathrm{H}^{+}+\mathrm{O}^{7+}
$$

and

$$
\mathrm{H}+\mathrm{He}^{2+} \rightarrow \mathrm{H}^{+}+\mathrm{He}^{+}
$$

and compared it with similar calculations done using the molecular approach by Harel et al 20 . The results are summarized in fig. 3. There is a sharp discrepancy in the behaviour for $v \rightarrow 0$, where the present model predicts an increasing cross section. At very low speed it is the concept itself of atomic distribution function which becomes questionable, and molecular aspects become important. Besides, quantum effects such as the discreteness of the energy levels also play a major role and are completely missed by this approach. In the higher velocity part, the present model underestimates the more accurate value by a factor 2 for process (18), but the error is much less, just $25 \%$, for process (19). These two ions have been chosen ad hoc: they correspond to values of the ratio $Z_{t} / Z_{p}=1 / 8$ and $1 / 2$ respectively. In the (I, Cs) test this ratio ranged from $\approx 1 / 12$ to $\approx 1 / 60$ depending upon the projectile charge. This means that in the former case the perturbation of the projectile on the electron distribution function is comparable to the (I, Cs) case, while in the latter it is much less. We expect the electron distribution function to be more and more perturbed as $Z_{t} / Z_{p} \rightarrow 0$. 


\section{SUMMARY AND CONCLUSIONS}

We have developed in this paper a very simple OBM for charge exchange. It exploits some features of the quantum mechanical version of the problem, thus differing from similar models which are solely classical. The agreement with experiment is much better than previous calculations where a comparison could be made. It is far from excellent, but reasons for the (partial) failure have been suggested.

As it stands, the model is well suited for one-optical-electron atoms (since it uses hydrogen-like wavefunctions), therefore we do expect that other classical OBM's can still work better in the many-electrons targets studied in previous experiments.

Some improvements are likely to be added to the present model: a possible line of investigation could be coupling the present method with a very simplified calculation of the evolution of the wavefunction, using quantum mechanics. From this one should not compute the $f$ as coming from a single state, but as a linear combination including also excited wavefunctions (the relative weights in the combination should be given by the quantum mechanical calculation). Work in this direction is currently underway.

\section{ACKNOWLEDGMENTS}

It is a pleasure to thank the staff at National Institute for Fusion Science (Nagoya), and in particular Prof. H. Tawara and Dr. K. Hosaka for providing the data of ref. [f] and for useful discussions about the subject. The referees through their suggestions and criticism have made the manuscript readable. 
[1] Hiroyuki A et al 1997 Fus Eng Design 34-35 785

[2] Hosaka K et al 1997 Phys Scr T73 273

[3] Kimura M et al 1995 J Phys B: At Mol Opt Phys 28 L643

[4] Hosaka K et al 1997 Fus Eng Design 34-35 781

[5] Niehaus A 1986 J Phys B: At Mol Phys 192925

[6] Nagano R, Yabana K, Tazawa T and Abe Y 1999 J Phys B: At Mol Opt Phys 32 L65

[7] Hosaka $\mathrm{K}$ et al Electron capture cross sections of low energy highly charged ions in collisions with alkali atoms, poster presented at the International Seminar on Atomic Processes in Plasmas held in Toki (Japan, 1999). To be published as a report of the National Institute for Fusion Science (NIFS)

[8] Ostrovsky V N 1995 J Phys B: At Mol Opt Phys 283901

[9] Ivanovski G, Janev R K, and Solov'ev E A 1995 J Phys B: At Mol Opt Phys 284799

[10] Ryufuku H, Sasaki K and Watanabe T 1980 Phys Rev A 21745

[11] Landau L D and Lifshitz E M 1977 Quantum Mechanics (Oxford, Pergamon) Eq. (48.5)

[12] Abrines R and Percival I C 1966 Proc Phys Soc 88861

[13] Sattin F and Bolzonella T 1998 Phys Scr 5853

[14] Samengo I 1998 Phys Rev A 582767

[15] Cohen J S 1985 J Phys B: At Mol Phys 181759

[16] Ema J et al 1999 At Data Nucl Data Tables 7257

[17] Eichenauer D, Grün N and Scheid W 1981 J Phys B: At Mol Phys 143929

[18] Hardie D J W and Olson R E 1983 J Phys B: At Mol Phys 161983

[19] Montemajor V J and Schiwietz G 1989 J Phys B: At Mol Opt Phys 222555

[20] Harel C, Jouin H and Pons B 1998 At Data Nucl Data Tables 68279 


\section{FIGURE CAPTIONS}

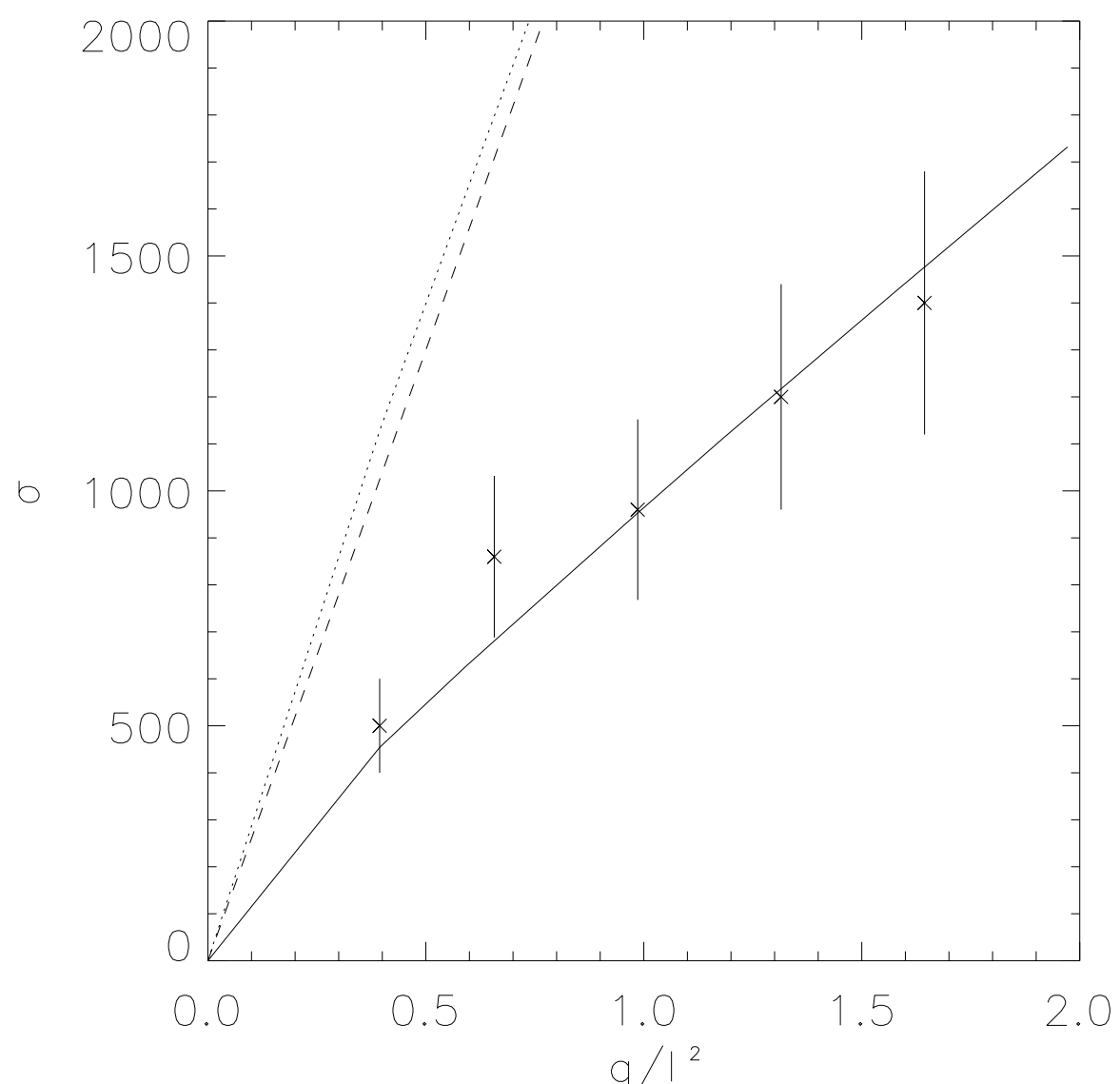

FIG. 1. Comparison between experimental datta and prediction from models for electron capture cross section of process (17). Stars, experiment with $20 \%$ error bar; dashed line, scaling law from Niehaus (Eq. E); dotted line, Ostrovsky's scaling law; solid line, scaling law from present model. $\sigma$ is in $10^{-20} \mathrm{~m}^{2}, I_{t}$ in eV. 


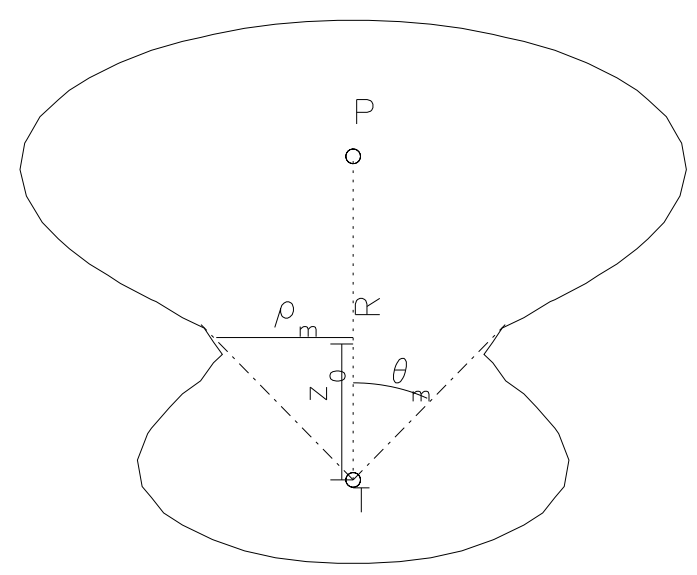

FIG. 2. Geometry of the scattering. $\mathbf{P}$ and $\mathbf{T}$ are the projectile and target nucleus respectively. The enveloping curve shows a section of the equipotential surface $U=E$, i.e., it is the border of the region classically accessible to the electron. $R$ is the internuclear distance. The parameter $\rho_{m}$ is the radius of the opening which joins the potential wells, $\theta_{m}$ the opening angle from $\mathbf{T} ; z_{0}$ is the position of the potential's saddle point.
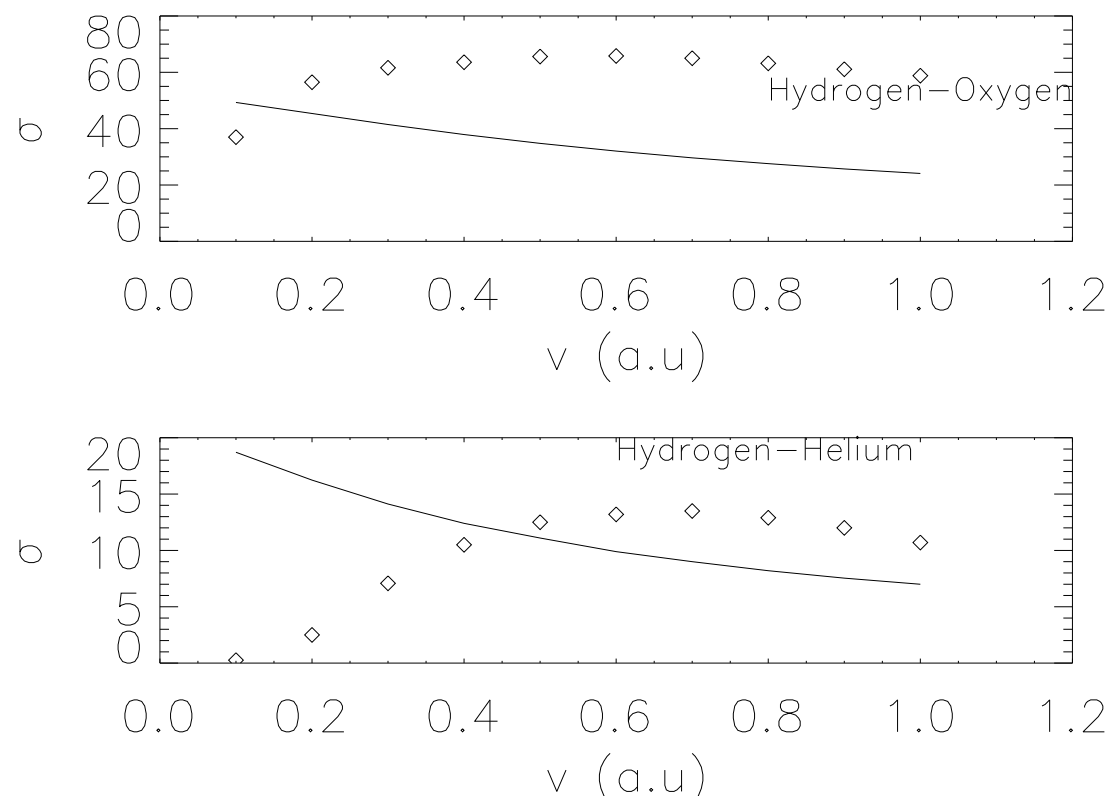

FIG. 3. Capture cross section versus impact velocity. Upper, $\mathrm{H}-\mathrm{O}^{8+}$ collisions; lower, $\mathrm{H}-\mathrm{He}^{2+}$ collisions. Diamonds, data from ref. 20; solid line, present model. 